\title{
Twenty year fitness trends in young adults and incidence of prediabetes and diabetes: the CARDIA study
}

\author{
Lisa S. Chow ${ }^{1}$ Andrew O. Odegaard ${ }^{2}$. Tyler A. Bosch ${ }^{1} \cdot$ Anne E. Bantle $^{1} \cdot$ Qi Wang $^{3}$. \\ John Hughes ${ }^{3}$ • Mercedes Carnethon ${ }^{4}$ - Katherine H. Ingram ${ }^{5}$ - Nefertiti Durant ${ }^{6}$. \\ Cora E. Lewis $^{7}$ - Justin Ryder ${ }^{8}$. Christina M. Shay ${ }^{9}$ - Aaron S. Kelly ${ }^{8}$. \\ Pamela J. Schreiner ${ }^{10}$
}

Received: 21 December 2015 / Accepted: 11 April 2016/Published online: 16 May 2016

(C) Springer-Verlag Berlin Heidelberg 2016

\begin{abstract}
Aims/hypothesis The prospective association between cardiorespiratory fitness (CRF) measured in young adulthood and middle age on development of prediabetes, defined as impaired fasting glucose and/or impaired glucose tolerance, or diabetes by middle age remains unknown. We hypothesised that higher fitness levels would be associated with reduced risk for developing incident prediabetes/diabetes by middle age.

Methods Participants were from the Coronary Artery Risk Development in Young Adults (CARDIA) study who were free from prediabetes/diabetes at baseline (year 0 [Y0]: 1985-1986). CRF was quantified by treadmill duration (converted to metabolic equivalents [METs]) at $\mathrm{Y} 0, \mathrm{Y} 7$ and $\mathrm{Y} 20$ and prediabetes/ diabetes status was assessed at $\mathrm{Y} 0, \mathrm{Y} 7, \mathrm{Y} 10, \mathrm{Y} 15, \mathrm{Y} 20$ and $\mathrm{Y} 25$. We use an extended Cox model with CRF as the primary timevarying exposure. BMI was included as a time-varying covariate. The outcome was development of either prediabetes or diabetes after Y0. Model 1 included age, race, sex, field centre, CRF and BMI. Model 2 additionally included baseline (Y0) smoking, energy intake, alcohol intake, education, systolic BP, BP medication use and lipid profile.
\end{abstract}

Lisa S. Chow

chow0007@umn.edu

1 Division of Diabetes, Endocrinology and Metabolism, Department of Medicine, University of Minnesota, MMC 101, 420 Delaware St SE, Minneapolis, MN 55455, USA

2 Department of Epidemiology, University of California Irvine, Irvine, CA, USA

3 Division of Biostatistics, University of Minnesota, Minneapolis, MN, USA

4 Department of Preventive Medicine, Northwestern University, Chicago, IL, USA
Results Higher fitness was associated with lower risk for developing incident prediabetes/diabetes (difference of 1 MET: HR 0.99898 [95\% CI 0.99861, 0.99940], $p<0.01$ ), which persisted (difference of 1 MET: HR 0.99872 [95\% CI $0.99840,0.99904], p<0.01]$ when adjusting for covariates. Conclusions/interpretation Examining participants who had fitness measured from young adulthood to middle age, we found that fitness was associated with lower risk for developing prediabetes/diabetes, even when adjusting for BMI over this time period. These findings emphasise the importance of fitness in reducing the health burden of prediabetes and diabetes.

Keywords Cardiorespiratory fitness $\cdot$ Diabetes $\cdot$ Prediabetes
Abbreviations
CARDIA Coronary Artery Risk Development in Young Adults
CRF Cardiorespiratory fitness
MET Metabolic equivalent

5 Department of Exercise Science and Sport Management, Kennesaw State University, Kennesaw, GA, USA

6 Department of Pediatrics, University of Alabama, Birmingham, AL, USA

7 Department of Preventive Medicine, University of Alabama, Birmingham, AL, USA

8 Department of Pediatrics, University of Minnesota, Minneapolis, MN, USA

9 Department of Nutrition, University of North Carolina at Chapel Hill, Chapel Hill, NC, USA

10 Division of Epidemiology, University of Minnesota, Minneapolis, MN, USA 
SBP Systolic BP

TG Triacylglycerol

\section{Introduction}

Americans are becoming increasingly obese and sedentary [1], which parallels the increasing epidemic of diabetes [2]. As physical activity is inversely associated with incident diabetes [3], the metabolic syndrome [4] and mortality [5], there is mounting interest in reducing morbidity and mortality by increasing physical activity and reducing sedentary time [6]. Although not equivalent due to genetics and behaviour effects, physical activity is directly related to cardiorespiratory fitness (CRF) [7]. Several prospective studies have observed higher CRF to be associated with lower future risk of coronary heart disease [8], the metabolic syndrome [9], diabetes [10] and total mortality [8].

Yet the benefits of higher CRF in young adulthood are not necessarily maintained across the lifespan. Compared with decreases in CRF over time, longitudinal improvement in CRF or maintenance of CRF through adulthood are associated with lower risk of hypertension [11], the metabolic syndrome [9], hyperlipidaemia [11], diabetes [10], cardiovascular mortality [12] and total mortality [13]. This literature, however, remains limited for several reasons. Much [12,13], but not all $[10,11]$, of the current literature on CRF change has been focused on men, with either varying duration between CRF assessments [11, 12], or a limited period ( $5-7$ years) between CRF assessments $[10,13]$. Although it is generally presumed that higher fitness is associated with lower rates of prediabetes, defined as impaired fasting glucose and/or impaired glucose tolerance, or diabetes prospectively, we present a unique opportunity to address this presumption using the Coronary Artery Risk Development in Young Adults (CARDIA) study (ClinicalTrials.gov: NCT00005130). The CARDIA study objectively documented fitness by using treadmill exercise testing to measure CRF at baseline (year 0 [Y0]: age 18-30 years), in early adulthood (Y7: age 25-37 years) and again at middle age (Y20: age 38-50 years), with documentation of incident prediabetes/diabetes over 25 years. This project extends a previous CARDIA analysis that reported that higher CRF in young adulthood and less CRF decline (over 7 years) reduce the 20 year risk for developing incident diabetes [10]. We hypothesised higher fitness levels, even after adjusting for changes in BMI, would be associated with reduced risk for developing incident prediabetes/diabetes.

\section{Methods}

Participants The CARDIA study is a prospective, multicentre, cohort study designed to investigate trends and determinants of coronary heart disease risk factors in young adults. Black and white women and men (ages 18-30 years at Y0) were recruited and examined in 1985-1986 from four US communities (Birmingham, AL; Chicago, IL; Minneapolis, MN and Oakland, CA) and balanced on age, race, sex and educational attainment as previously described [14]. A total of 5,115 participants were enrolled at baseline (Y0) with follow-up examinations at 2, 5, 7, 10, 15, 20 and 25 years after baseline, with retention of $91 \%, 86 \%, 81 \%, 79 \%, 74 \%, 72 \%$ and $72 \%$ of the surviving cohort, respectively. All participants provided written informed consent. The study was approved by the Institutional Review Boards from each participating institution.

For this particular study, we excluded participants that did not have baseline treadmill results ( $n=69$ excluded) or diabetes assessment at baseline (additional $n=80$ excluded). We also excluded the CARDIA participants who had a diagnosis of prediabetes at Y0 (additional $n=105$ excluded) or diabetes (additional $n=28$ excluded) or did not have at least one follow-up visit documenting prediabetes/diabetes status (additional $n=460$ excluded). After application of exclusion criteria, 4,373 CARDIA participants were available for analysis. Participants who did not develop prediabetes/ diabetes by the time of their last available examination visit were censored.

CRF A symptom-limited graded treadmill exercise testing using a modified Balke protocol [15] was used to assess CRF in the CARDIA study. The test protocol was designed to assess maximal, symptom-limited performance. After baseline measurement of pulse, BP and ECG, the participant started the protocol. The protocol consisted of nine stages ( $2 \mathrm{~min} / \mathrm{stage}$, maximum $18 \mathrm{~min} / \mathrm{test}$ ) of progressively increasing difficulty, with the first six stages generally performed by walking. Stage 1 was $4.8 \mathrm{~km} / \mathrm{h}$ at $2 \%$ grade (4.1 metabolic equivalents [METs]), progressing to stage 9 at $9.0 \mathrm{~km} / \mathrm{h}$ at $25 \%$ grade (19.0 METs). The exercise test was terminated either due to fatigue $(82.5 \%)$, shortness of breath $(7.5 \%)$, abnormal ECG response (1\%), medical reasons (5.3\%), participant refusal $(1.6 \%)$ or completion of the entire protocol $(0.2 \%)$ [15]. As previously described, treadmill duration as assessed by the Balke protocol has been shown to be highly predictive $(r=-0.94)$ of maximum oxygen uptake $\left(\dot{V}_{2 \max }\right)$ [16]. Treadmill duration was converted to equivalent METs using a previously published conversion formula [15].

In the CARDIA cohort, the treadmill test was performed at Y0, Y7 and Y20. Because of an identified systemic protocol violation at $Y 7$ at one site [17], the treadmill results from $Y 0$, Y7 and Y20 were used when appropriate (three sites). In the extended Cox model, fitness prior to $\mathrm{Y} 7$ was represented by Y0 CRF (all sites), fitness between Y7 and Y20 was represented by Y7 CRF (for the three valid sites: $n=2,415$, otherwise Y0 CRF was used for the remaining site: $n=1,195$ ) and 
fitness between Y20 and Y25 was represented by Y20 CRF (all sites).

Ascertainment of prediabetes and diabetes At Y0, Y7, Y10, Y15, Y20 and Y25, prediabetes and diabetes status were assessed. The outcome was development of either prediabetes or diabetes (described as prediabetes/diabetes) after Y0. Once a participant developed either documented prediabetes or diabetes after Y0, they were considered to have an event and were subsequently censored. The outcome for the extended Cox model was time to development of incident prediabetes/diabetes. This was calculated as the time to the CARDIA follow-up visit where the diagnosis was made. Participants were classified as having prediabetes if they met any of the following criteria: fasting glucose 5.6-6.9 mmol/l; 2 h OGTT glucose 7.8-11.1 mmol/1 or $\mathrm{HbA}_{1 \mathrm{c}} 5.7-6.4 \%$ (39-46 mmol/mol IFCC). Participants were classified as having diabetes if any of the following criteria was met: fasting glucose $\geq 7 \mathrm{mmol} / \mathrm{l}$; use of medications for diabetes treatment; $2 \mathrm{~h}$ GTT $\geq 11.1 \mathrm{mmol} / 1$ or $\mathrm{HbA}_{1 \mathrm{c}} \geq 6.5 \%$ (48 $\mathrm{mmol} / \mathrm{mol}$ IFCC) [10]. Serum glucose concentrations were measured using the hexokinase method.

Covariates Covariates were selected in our analysis due to their clinical relevance and association with diabetes and/or CRF. Relevant covariates measured at Y0 included age, sex, race, field centre, BMI, smoking (current/former/never), energy intake ( $\mathrm{kJ} /$ day), alcohol intake ( $\mathrm{ml} /$ day) and education level (highest year of school completed). To quantify physical activity (reported as exercise units [EU]), the CARDIA physical activity history questionnaire was used, which was an interviewer-based self-report of duration and intensity of participation in 13 categories of exercise over the previous 12 months [18]. As a reference, $300 \mathrm{EU}$ approximates to $150 \mathrm{~min}$ of moderate-intensity activity (3-5 METs, with 1 MET defined as energy expenditure at rest or $3.5 \mathrm{ml} \mathrm{O}_{2} / \mathrm{kg}$ ) per week or 30 min of moderate-intensity activity 5 days per week [19]. These covariates were measured by trained and certified staff, using standardised protocols across field centres and examinations with quality control monitoring [14, 20]. Age, race and sex were confirmed during the clinic visits. Diet was measured using an interviewer-administrated, validated, semi-quantitative, food frequency questionnaire [21].

Body weight was measured with light clothing to the nearest $0.09 \mathrm{~kg}$, body height without shoes was measured to the nearest $0.5 \mathrm{~cm}$ and BMI was calculated from these measures in units of $\mathrm{kg} / \mathrm{m}^{2}$ [20]. Because time-varying BMI was included in the analysis, we used Y0 BMI as a predictor for outcomes between $\mathrm{Y} 0$ and $\mathrm{Y} 7, \mathrm{Y} 7 \mathrm{BMI}$ as a predictor for outcomes between Y7 and Y10, Y10 BMI for outcomes between Y10 and Y15, Y15 BMI for outcomes between Y15 and Y20 and Y20 BMI for outcomes between Y20 and Y25.

We also used several cardiometabolic risk factors (systolic BP $[\mathrm{SBP}]$, medication treatment of hypertension, HDL- cholesterol and LDL-cholesterol) in the analysis. Cardiometabolic risk factors were measured using standardised protocols across field centres and examinations with quality control monitoring $[14,20]$. BP was measured three times after a 5 min rest, using a Hawksley random zero sphygmomanometer (WA Baum Company, Copaigue, NY, USA) on the right arm of the seated participant. The average of the last two measures of three was used for Y0 SBP [20]. Fasting plasma blood samples were sent to the Northwest Lipid Research Laboratories, University of Washington (Seattle, WA, USA) for lipid determination. Total cholesterol and triacylglycerol (TG) were measured enzymatically [22]. HDL-cholesterol was determined after dextran sulfate-magnesium chloride precipitation [23] and LDL-cholesterol was calculated using the Friedewald equation [24].

Statistical analysis In Table 1, participant Y0 characteristics are presented by whether they developed incident prediabetes/ diabetes by Y25, using means and SD for continuous variables and percentages for categorical variables. Two-sample $t$ tests and $\chi^{2}$ tests were performed for continuous variables and categorical variables, respectively, to compare the two groups.

Modelling was performed using an extended Cox regression analysis, with inclusion of fitness and BMI as timevarying covariates. The outcome was incidence of prediabetes or diabetes as documented at Y7, Y10, Y15, Y20 or Y25. Model 1 included age, race, sex, field centre, time-dependent CRF and time-dependent BMI. Model 2 additionally included Y0 lifestyle factors (smoking, energy intake, alcohol intake, education, SBP, medication for hypertension, LDLcholesterol and HDL-cholesterol). For both Model 1 and Model 2, we examined whether there was significant interaction between race and sex. As interaction between race and sex was not significant, this was therefore not included in Model 1 and Model 2. The extended Cox regression modelling approach eliminates the need to test for proportional hazards because the models are not restricted to baseline values of the exposures of interest. All analyses were performed using Statistical Analysis Software (version 9.3; SAS Institute, Cary, NC, USA).

\section{Results}

Subject characteristics Compared with CARDIA participants who did not develop prediabetes or diabetes by Y25 (Table 1), the participants who developed prediabetes or diabetes by $\mathrm{Y} 25$ tended to be older, black race and male sex, were more likely to take blood pressure medication, more likely to smoke, have higher weight, BMI, waist circumference, SBP, LDL, daily alcohol intake and daily energy intake, and show a decline in treadmill duration over 20 years. Compared with 
Table 1 Baseline (Y0) characteristics of CARDIA participants comparing those who did and did not develop incident prediabetes/diabetes by middle age (Y25)

\begin{tabular}{|c|c|c|c|}
\hline Characteristic & $\begin{array}{l}\text { Absence of } \\
\text { prediabetes/diabetes } \\
\text { by Y25 } \\
(n=1,927)\end{array}$ & $\begin{array}{l}\text { Development of incident } \\
\text { prediabetes/diabetes by Y25 } \\
(n=2,446)\end{array}$ & $p$ value \\
\hline Age (years) & $24.4 \pm 3.7$ & $25.2 \pm 3.6$ & $<0.01$ \\
\hline Race, white & $1,090(56.6)$ & $1,105(45.2)$ & $<0.01$ \\
\hline Sex, female & $1,213(63)$ & $1,200(49.1)$ & $<0.01$ \\
\hline Weight $(\mathrm{kg})$ & $66.9 \pm 14.0$ & $74.0 \pm 16.6$ & $<0.01$ \\
\hline BMI $\left(\mathrm{kg} / \mathrm{m}^{2}\right)$ & $23.2 \pm 4.2$ & $25.3 \pm 5.2$ & $<0.01$ \\
\hline Waist circumference $(\mathrm{cm})$ & $75.0 \pm 9.7$ & $79.9 \pm 11.7$ & $<0.01$ \\
\hline SBP (mmHg) & $109.0 \pm 10.4$ & $112 \pm 10.9$ & $<0.01$ \\
\hline No medications for BP & $1,895(98.4)$ & $2,378(97.3)$ & 0.02 \\
\hline LDL-cholesterol (mmol/1) & $2.7 \pm 0.8$ & $2.9 \pm 0.8$ & $<0.01$ \\
\hline HDL-cholesterol (mmol/l) & $1.4 \pm 0.3$ & $1.3 \pm 0.3$ & $<0.01$ \\
\hline Y0 Education level, $>12$ years & $1,208(62.7)$ & $1,501(60.4)$ & 0.36 \\
\hline Cigarette smoking & & & 0.01 \\
\hline Never & $1,123(58.6)$ & $1,380(56.8)$ & \\
\hline Former & $275(14.4)$ & $301(12.4)$ & \\
\hline Current & $518(27.0)$ & $748(30.8)$ & \\
\hline Alcohol (ml/day) & $10.7 \pm 20.4$ & $12.5 \pm 21.9$ & $<0.01$ \\
\hline Physical activity intensity score (EU) & $418 \pm 288$ & $421 \pm 304$ & 0.77 \\
\hline Total energy intake (mJ/day) & $11.7 \pm 6.3$ & $12.6 \pm 7.6$ & $<0.01$ \\
\hline Total energy intake (kcal/day) & $2,791 \pm 1,493$ & $3,021 \pm 1,809$ & $<0.01$ \\
\hline Y0 treadmill duration, all sites (s) & $590 \pm 180$ & $575 \pm 187$ & $<0.01$ \\
\hline Y0 treadmill energy expenditure, all sites (METs) & $11.8 \pm 2.9$ & $11.5 \pm 3.0$ & $<0.01$ \\
\hline Y7 treadmill duration, three sites (s) & $558 \pm 174^{\mathrm{a}}$ & $527 \pm 177^{\mathrm{b}}$ & $<0.01$ \\
\hline Y7 treadmill energy expenditure, three sites (METs) & $11.3 \pm 2.7^{\mathrm{a}}$ & $10.8 \pm 2.8^{\mathrm{b}}$ & $<0.01$ \\
\hline Y20 treadmill duration, all sites (s) & $460 \pm 158^{\mathrm{c}}$ & $407 \pm 159^{\mathrm{d}}$ & $<0.01$ \\
\hline Y20 treadmill energy expenditure, all sites (METs) & $9.8 \pm 2.5^{\mathrm{c}}$ & $8.9 \pm 2.6^{\mathrm{d}}$ & $<0.01$ \\
\hline $\mathrm{Y} 20-\mathrm{Y} 0$ change in treadmill duration (s) & $-148 \pm 135^{\mathrm{c}}$ & $-182 \pm 134^{\mathrm{d}}$ & $<0.01$ \\
\hline Participants who increased CRF over 20 years & $119(10.8)$ & $111(6.8)$ & $<0.01$ \\
\hline
\end{tabular}

Data are means \pm SD for continuous variables or number (\%) for categorical variables, $N=4,373$

${ }^{\mathrm{a}} n=1,018$

${ }^{\mathrm{b}} n=1,397$

${ }^{\mathrm{c}} n=1,097$

${ }^{\mathrm{d}} n=1,632$

CARDIA participants who did not develop prediabetes or diabetes by Y25, the participants who developed prediabetes or diabetes by Y25 group had lower Y0 CRF (as quantified by $\mathrm{Y} 0$ treadmill duration and converted to $\mathrm{Y} 0$ treadmill energy expenditure in METs), lower HDL-cholesterol, lower Y7 CRF (as quantified by $\mathrm{Y} 7$ treadmill duration and converted to $\mathrm{Y} 7$ treadmill energy expenditure in METs), lower Y20 CRF (as quantified by Y20 treadmill duration and converted to Y20 treadmill energy expenditure in METs) and a lower proportion who increased their CRF over 20 years. There was no difference in educational level or physical activity level between the two groups.

Self-reported physical activity obtained by questionnaire was related to objectively measured CRF: Y0 physical activity correlated with Y0 CRF (METs) $(r=0.39, p<0.01$, all sites used).
Y0 CRF (METs) correlated with Y7 CRF (METs) $(r=0.76$, $p<0.01,3$ sites used) and Y0 CRF (METs) correlated with Y20 CRF (METs) $(r=0.69, p<0.01$, all sites used).

\section{Association of CRF with development of prediabetes/} diabetes by Y25 In this study $(N=4,373), 1,927(44.1 \%)$ participants did not develop either prediabetes or diabetes by Y25. By Y25, 1,941 (44.5\%) participants developed prediabetes and $505(11.5 \%)$ participants developed diabetes.

We examined associations between CRF (METs) with the development of prediabetes or diabetes by Y25 (Table 2). While taking the time-varying aspect of BMI into account, we observed that higher CRF (per 1 MET increment) was associated with lower risk for developing incident prediabetes/diabetes 
Table 2 Association of CRF change from young adulthood to middle age with development of prediabetes/diabetes by middle age

\begin{tabular}{|c|c|c|c|c|}
\hline \multirow[t]{2}{*}{$\mathrm{CRF}$} & \multicolumn{2}{|l|}{ Model $1^{\mathrm{a}}$} & \multicolumn{2}{|l|}{ Model $2^{b}$} \\
\hline & HR $(95 \%$ CI $)$ & $p$ value & $\mathrm{HR}(95 \% \mathrm{CI})$ & $p$ value \\
\hline CRF (per 1 MET increment) & $0.99898(0.99861,0.99940)$ & $<0.01$ & $0.99872(0.99840,0.99904)$ & $<0.01$ \\
\hline
\end{tabular}

(0.99898 [95\% CI 0.99861, 0.99940]), which persisted (0.99872 [95\% CI 0.99840, 0.99904]) after accounting for Y0 lifestyle factors (smoking, energy intake, alcohol intake, education) SBP, medication for blood pressure, LDL-cholesterol levels and HDL-cholesterol levels.

\section{Discussion}

As the benefit of high levels of fitness in young adulthood is well documented in current literature, this study evaluated the association between prospectively measured CRF over 20 years on incident prediabetes or diabetes by middle age. We found that CRF was associated with lower risk for developing prediabetes/diabetes, even when adjusting for BMI over this time period. These findings emphasise the importance of continued lifelong fitness in reducing the risk for developing prediabetes/diabetes.

Given high baseline levels of fitness, the prospective health benefits are well established $[8,13]$, with CRF mitigating the risk for developing impaired fasting glucose and type 2 diabetes even in obese participants [25]. The natural extension of this literature is to evaluate the metabolic impact of fitness, objectively quantified prospectively. By leveraging the unique design of CARDIA, this study complements and extends the current literature [10-13] by examining the association of changes in fitness over 20 years, from young adulthood to middle age, on developing prediabetes/diabetes, while considering concurrent changes in BMI.

There are several mechanisms by which continued fitness may reduce the risk for prediabetes and diabetes. Twenty weeks of supervised exercise training has been shown to improve the components of the metabolic syndrome, such as reduced TG, fasting glucose, waist circumference and improved HDLcholesterol levels[4]. One mechanism may arise from the effect of exercise on visceral fat. Compared with subcutaneous fat, accumulation of visceral fat is associated with more adverse metabolic risk factors [26] and higher risk of diabetes [27]. With ageing, muscle mass declines[28, 29] and visceral fat increases $[30,31]$. Since exercise training, depending on type, duration and intensity, reduces visceral fat and increases muscle mass [32, 33], this may be a significant protective mechanism. In addition, type 2 diabetes is associated with low-grade systemic inflammation [34]. Potentially, another mechanism may arise from the anti-inflammatory effect of exercise [35, 36]. Shortterm $(20$ weeks) supervised exercise training has been shown to reduce C-reactive protein (median $1.34 \mathrm{mg} / \mathrm{l}$ reduction) in participants with the highest level of C-reactive protein $(>3.0 \mathrm{mg} / \mathrm{l})$ prior to training [37]. In the absence of weight change, smaller clinical studies have shown that exercise training improves fitness, as measured by $\dot{V} \mathrm{O}_{2 \max }$, and insulin sensitivity, as measured by the gold standard hyperinsulinaemiceuglycaemic clamp $[38,39]$. Our findings support the longterm implications of these short-term mechanistic findings.

This study is clinically relevant as it provides evidence to support the commonly accepted dogma that fitness is beneficial in reducing the risk for prediabetes/diabetes. As this benefit remained significant even when adjusting for BMI, exercise programmes remain critically important for reducing the adverse health impact of prediabetes, diabetes, and their associated complications. From a clinical standpoint, the amount of physical activity required to increase an individual's fitness by $1 \operatorname{MET}\left(3.5 \mathrm{ml} \mathrm{O}_{2} \mathrm{~kg}^{-1} \mathrm{~min}^{-1}\right)$ depends on multiple factors, which include the age, health and baseline activity of the individual as well as the duration, intensity and form of the exercise. As a reference, the STRRIDE-AT/RT (Studies Targeting Risk Reduction Interventions through Defined Exercise-Aerobic Training and/or Resistance Training) study found that sedentary obese/overweight men and women (mean age 51 years, baseline $\dot{V} \mathrm{O}_{2 \max } 27.1 \mathrm{ml} \mathrm{O}_{2} \mathrm{~kg}^{-1}$ $\min ^{-1}$ ) increased $\dot{V} \mathrm{O}_{2 \max }$ by $3.9 \mathrm{ml} \mathrm{O}_{2} \mathrm{~kg}^{-1} \mathrm{~min}^{-1}$ with 8 months of aerobic training $(0.06 \mathrm{~mJ}(14 \mathrm{kcal}) / \mathrm{kg}$ per week $)$ and by $4.1 \mathrm{ml} \mathrm{O}_{2} \mathrm{~kg}^{-1} \mathrm{~min}^{-1}$ with 8 months of combined aerobic and resistance training $(0.06 \mathrm{~mJ}(14 \mathrm{kcal}) / \mathrm{kg}$ per week +180 min resistance training per week) [40]. The high rate of incident prediabetes in the CARDIA cohort additionally highlights the preventative aspect of fitness in reducing prediabetes, which predicts and precedes the development of diabetes.

This study has several strengths. It focuses on an important demographic - young adults (age 18-30 years) followed prospectively through to middle age (age 43-55 years). It also 
capitalises on several key features of CARDIA, specifically the prospective objective measurement of CRF by treadmill testing, balanced cohort in terms of sex, race and educational attainment, outcome measurements using standardised protocols with rigorous quality control and frequent in-person follow-up visits to ascertain incident prediabetes/diabetes. Consequently, the ability to quantify time to event, on the scale of the CARDIA study, is a study strength. Because of its objective measures of CRF over several time points and standardised long-term follow-up, CARDIA is a unique resource with which to address the relative association of prospective measures of fitness with prospective development of incident prediabetes/diabetes.

This study also has several limitations. CRF is not equivalent to physical activity and has a significant genetic component [41]. However, physical activity has been shown to have a dose-response relationship to cardiovascular CRF, with physical activity positively correlated with CRF [7]. We also saw a modest correlation between baseline physical activity and fitness in our data. In CARDIA, CRF was measured by treadmill duration and did not account for weight, with the possibility that reduced treadmill duration is not necessarily due to reduced CRF but rather weight gain. We adjusted for this possibility by including time-varying BMI in our modelling. We also acknowledge that participants, particularly in the group that developed prediabetes/diabetes by Y25, may terminate the treadmill test due to reasons other than reaching their full physical capability. Although this may reduce the quantification of CRF by treadmill duration, early test termination, even if participants are physically capable, still remains an adverse prognostic indicator. We also acknowledge the presence of the 'healthy cohort' phenomenon, such that participants who can be followed up are generally healthier than those lost to followup. This limitation would tend to underestimate the observed associations by excluding those more likely to develop prediabetes/diabetes and less likely to maintain fitness levels.

In summary, by examining participants who had fitness measured from young adulthood to middle age, we found that fitness was associated with lower risk for developing prediabetes/diabetes, even when adjusting for BMI over this time period. These findings emphasise the importance of fitness in reducing the health burden of prediabetes/diabetes.

Acknowledgements The authors thank C. Basu from the Division of Epidemiology and Community Health, University of Minnesota, for creating the analytic dataset for analysis. The authors acknowledge the assistance of M. Sarzynski at the Pennington Biomedical Research Center for his critical review of the manuscript. The authors thank the staff and participants of the CARDIA study for their important contributions.

A portion of this data was presented at the ADA 75th Scientific Sessions, San Francisco, June 2015.

Contribution statement LSC, AOO, MC and PJS designed the research. MC, AOO and PJS provided essential data for analysis. LSC,
QW, JH and PJS analysed the data. All authors provided substantial contributions to the analysis and interpretation of the data LSC, QW, JH and PS wrote the manuscript and all authors revised it critically for important intellectual content. All authors have read and approved the final manuscript. LSC has primary responsibility for the final content.

Duality of interest The authors declare that there is no duality of interest associated with this manuscript.

Funding The CARDIA study is supported by contracts HHSN268201300025C, HHSN268201300026C, HHSN268201300027C, HHSN $268201300028 \mathrm{C}$, HHSN $268201300029 \mathrm{C}$ and HHSN268200900041C from the National Heart, Lung, and Blood Institute (NHLBI), the Intramural Research Programme of the National Institute on Aging (NIA) and an intra-agency agreement between NIA and NHLBI (AG0005). The content is solely the responsibility of the authors and does not necessarily represent the official views of the NIA, NHLBI or National Institutes of Health.

\section{References}

1. Manson JE, Skerrett PJ, Greenland P, VanItallie TB (2004) The escalating pandemics of obesity and sedentary lifestyle. A call to action for clinicians. Arch Intern Med 164:249-258

2. CDC (2014) Rate per 100 of civilian, noninstitutionalized population with diagnosed diabetes, by age, United States, 1980-2011. Available from www.cdc.gov/diabetes/statistics/prev/national/ figbyage.htm. Accessed 30 April 2015

3. Hu G, Lindstrom J, Valle TT et al (2004) Physical activity, body risk of type 2 diabetes in patients with normal or impaired glucose regulation. Arch Intern Med 164:892-896

4. Katzmarzyk PT, Leon AS, Wilmore JH et al (2003) Targeting the metabolic syndrome with exercise: evidence from the HERITAGE Family Study. Med Sci Sports Exerc 35:1703-1709

5. Paffenbarger RS, Hyde RT, Wing AL, Hsieh CC (1986) Physical activity, all-cause mortality, and longevity of college alumni. $\mathrm{N}$ Engl J Med 314:605-613

6. Kaminsky LA, Arena R, Beckie TM et al (2013) The importance of cardiorespiratory fitness in the United States: the need for a national registry: a policy statement from the American Heart Association. Circulation 127:652-662

7. Church TS, Earnest CP, Skinner JS, Blair SN (2007) Effects of different doses of physical activity on cardiorespiratory fitness among sedentary, overweight or obese postmenopausal women with elevated blood pressure: a randomized controlled trial. JAMA 297:2081-2091

8. Blair SN, Kampert JB, Kohl HW et al (1996) Influences of cardiorespiratory fitness and other precursors on cardiovascular disease and all-cause mortality in men and women. JAMA 276:205-210

9. Carnethon MR, Gidding SS, Nehgme R, Sidney S, Jacobs DR, Liu $\mathrm{K}$ (2003) Cardiorespiratory fitness in young adulthood and the development of cardiovascular disease risk factors. JAMA 290:30923100

10. Carnethon MR, Sternfeld B, Schreiner PJ et al (2009) Association of 20-year changes in cardiorespiratory fitness with incident type 2 diabetes: the coronary artery risk development in young adults (CARDIA) fitness study. Diabetes Care 32:1284-1288

11. Lee DC, Sui X, Church TS, Lavie CJ, Jackson AS, Blair SN (2012) Changes in fitness and fatness on the development of 
cardiovascular disease risk factors hypertension, metabolic syndrome, and hypercholesterolemia. J Am Coll Cardiol 59:665-672

12. Lee DC, Sui X, Artero EG et al (2011) Long-term effects of changes in cardiorespiratory fitness and body mass index on all-cause and cardiovascular disease mortality in men: the Aerobics Center Longitudinal Study. Circulation 124:2483-2490

13. Blair SN, Kohl HW, Barlow CE, Paffenbarger RS, Gibbons LW, Macera CA (1995) Changes in physical fitness and all-cause mortality. A prospective study of healthy and unhealthy men. JAMA 273:1093-1098

14. Friedman GD, Cutter GR, Donahue RP et al (1988) CARDIA: study design, recruitment, and some characteristics of the examined subjects. J Clin Epidemiol 41:1105-1116

15. Sidney S, Haskell WL, Crow R et al (1992) Symptom-limited graded treadmill exercise testing in young adults in the CARDIA study. Med Sci Sports Exerc 24:177-183

16. Pollock ML, Foster C, Schmidt D, Hellman C, Linnerud AC, Ward A (1982) Comparative analysis of physiologic responses to three different maximal graded exercise test protocols in healthy women. Am Heart J 103:363-373

17. Sidney S, Sternfeld B, Haskell WL, Quesenberry CP Jr, Crow RS, Thomas RJ (1998) Seven-year change in graded exercise treadmill test performance in young adults in the CARDIA study. Cardiovascular Risk Factors in Young Adults. Med Sci Sports Exerc 30:427-433

18. Jacobs DR, Hahn LP, Haskell WL, Pirie P, Sidney S (1989) Validity and reliability of short physical activity history: cardia and the Minnesota heart health program. J Cardiopulm Rehabil Prev 9: 448-459

19. Parker ED, Schmitz KH, Jacobs DR, Dengel DR, Schreiner PJ (2007) Physical activity in young adults and incident hypertension over 15 years of follow-up: the CARDIA study. Am J Public Health 97:703-709

20. Cutter GR, Burke GL, Dyer AR et al (1991) Cardiovascular risk factors in young adults. The CARDIA baseline monograph. Control Clin Trials 12:1S-77S

21. Liu K, Slattery M, Jacobs D et al (1994) A study of the reliability and comparative validity of the CARDIA dietary history. Ethn Dis 4:15-27

22. Warnick GR (1986) Enzymatic methods for quantification of lipoprotein lipids. Methods Enzymol 129:101-123

23. Warnick GR, Benderson J, Albers JJ (1982) Dextran sulfate-Mg2+ precipitation procedure for quantitation of high-density-lipoprotein cholesterol. Clin Chem 28:1379-1388

24. Friedewald WT, Levy RI, Fredrickson DS (1972) Estimation of the concentration of low-density lipoprotein cholesterol in plasma, without use of the preparative ultracentrifuge. Clin Chem 18:499 502

25. Lee D, Sui X, Church T, Lee I, Blair S (2009) Associations of cardiorespiratory fitness and obesity with risks of impaired fasting glucose and type 2 diabetes in men. Diabetes Care 32:257-262
26. Fox CS, Massaro JM, Hoffmann U et al (2007) Abdominal visceral and subcutaneous adipose tissue compartments - association with metabolic risk factors in the Framingham Heart Study. Circulation 116:39-48

27. Neeland IJ, Turer AT, Ayers CR et al (2012) Dysfunctional adiposity and the risk of prediabetes and type 2 diabetes in obese adults. JAMA 308:1150-1159

28. Doherty TJ (2003) Aging and sarcopenia. J Appl Physiol 95:17171727

29. Chow LS, Nair KS (2005) Sarcopenia of male aging. Endocrinol Metab Clin N Am 34:833-852

30. Borkan GA, Hults DE, Gerzof SG, Robbins AH, Silbert CK (1983) Age changes in body composition revealed by computed tomography. J Gerontol 38:673-677

31. Enzi G, Gasparo M, Biondetti PR, Fiore D, Semisa M, Zurlo F (1986) Subcutaneous and visceral fat distribution according to sex, age, and overweight, evaluated by computed tomography. Am J Clin Nutr 44:739-746

32. Schwartz RS, Shuman WP, Larson V et al (1991) The effect of intensive endurance exercise training on body fat distribution in young and older men. Metab Clin Exp 40:545-551

33. Wilmore JH, Despres JP, Stanforth PR et al (1999) Alterations in body weight and composition consequent to $20 \mathrm{wk}$ of endurance training: the HERITAGE Family Study. Am J Clin Nutr 70:346352

34. Pradhan AD, Manson JE, Rifai N, Buring JE, Ridker PM (2001) Creactive protein, interleukin 6 , and risk of developing type 2 diabetes mellitus. JAMA 286:327-334

35. Petersen AMW, Pedersen BK (2005) The anti-inflammatory effect of exercise. J Appl Physiol 98:1154-1162

36. Geffken DF, Cushman M, Burke GL, Polak JF, Sakkinen PA, Tracy RP (2001) Association between physical activity and markers of inflammation in a healthy elderly population. Am J Epidemiol 153: 242-250

37. Lakka TA, Lakka HM, Rankinen T et al (2005) Effect of exercise training on plasma levels of C-reactive protein in healthy adults: the HERITAGE Family Study. Eur Heart J 26:2018-2025

38. Perseghin G, Price TB, Petersen KF et al (1996) Increased glucose transport-phosphorylation and muscle glycogen synthesis after exercise training in insulin-resistant subjects. N Engl J Med 335: 1357-1362

39. Ostergard T, Andersen JL, Nyholm B et al (2006) Impact of exercise training on insulin sensitivity, physical fitness, and muscle oxidative capacity in first-degree relatives of type 2 diabetic patients. Am J Physiol Endocrinol Metab 290:E998-E1005

40. AbouAssi H, Slentz CA, Mikus CR et al (2015) The effects of aerobic, resistance, and combination training on insulin sensitivity and secretion in overweight adults from STRRIDE AT/RT: a randomized trial. J Appl Physiol 118:1474-1482

41. Bouchard C, Sarzynski MA, Rice TK et al (2011) Genomic predictors of the maximal $\mathrm{O} 2$ uptake response to standardized exercise training programs. J Appl Physiol 110:1160-1170 